\title{
La imperiosa necesidad del bacalao: Puerto Rico y Terranova en la Ecología-Mundo Manuel Valdes Pizzini*
}

\section{RESUMEN}

Este artículo explora, por medio del examen y análisis de estudios antropológicos, históricos y de las fuentes documentales de varios archivos, la relación entre Puerto Rico y Terranova, como consumidores y productores de pescado salado, respectivamente. El bacalao fue un alimento barato usado para sostener a la fuerza de trabajo esclavizada y libre en la larga duración caribeña y trasatlántica. El desarrollo del capitalismo, según Jason Moore, ha dependido de la expansión de las fronteras productivas (zonas de explotación de mano de obra y recursos) y de la extracción y consumo de insumos baratos. Para ello, ha dependido de la extracción de un excedente ecológico basado, en este caso, en la apropiación del trabajo no remunerado de mujeres y niños. El bacalao se produjo en unas condiciones de explotación y trabajo no remunerado de hombres, mujeres y niños en Terranova, para alimentar a familias pobres en Puerto Rico.

El objetivo principal de este trabajo consiste aplicar esos planteamientos teóricos de Moore, en el análisis de esas dos formaciones sociales, que han sido colonias de poderes imperiales; ambas vinculadas en una Ecología-Mundo basada en la producción y el consumo del bacalao; un proceso mediado por las clases mercantiles de ambos territorios. Las islas caribeñas estuvieron vinculadas a ese comercio trasatlántico (que incluía esclavos, melaza, rones y maderas) a través de intercambios con Terranova y Nueva Inglaterra, desde el siglo XVI. Los siglos XIX y XX, con el auge de la producción azucarera en Puerto Rico, fueron determinantes en establecer ese patrón de consumo y Terranova fue uno de los principales productores y suplidores de ese producto a Puerto Rico y el Caribe, manteniendo una estrategia de expansión de la frontera productiva, saliendo de la pesca costera para pescar en los grandes bancos, mediante un proceso sistemático de acumulación e inversión de capital, articulado por innovaciones en las artes de pesca y las embarcaciones.

Este artículo sugiere que la suma de las importaciones por los países del Caribe contribuyó al eventual colapso de los abastos pesqueros de Terranova. Este trabajo es un primer paso en el análisis de la profunda relación entre el capital y la naturaleza y un paso en la exploración de como esas relaciones trasatlánticas fueron configurando el trabajo y la naturaleza en dos extremos del océano. Cuando se escriba la historia de las poblaciones de peces y los abastos pesqueros de Terranova, habrá que contar con la participación de Puerto Rico, y su rol en el proceso de intensificación de la producción, es decir, de la expansión de la frontera vertical y de la horizontal de la producción.

\section{Palabras Clave}

Terranova; Puerto Rico; bacalao; fronteras productivas; excedente ecológico.

\section{TitLE}

The Urgent Need for Cod: Puerto Rico and Newfoundland in the World-Ecology

\section{EXTENDED Abstract}

Puerto Rico and Newfoundland are located at two extremes of the Atlantic Ocean, the former in the Caribbean Sea and the latter in the North Sea of the American continent. Both formations - former colonies from Spain and Great Britain-have been linked through the production and consumption of salted fish (that is, cod and other members of the Gadidae family)
* ManuelVALDÉS PIZZINI,

Doctor en Antropología e investigador del Centro Interdisciplinario de Estudios del Litoral en la Universidad de Puerto Rico en Mayagüez. Estudia los aspectos sociales de las pesquerías locales y la relación histórica entre los humanos y la naturaleza, con especial atención a los ecosistemas marinos y costeros. Contacto: manuel. valdes@upr.edu

Recibido:

16.09.2020

Aceptado:

|6.04.202|

DOI:

https://doi.org// 0.15366/relacionesinternacionales2021.47.008

Formato de citación recomendado:

VALDÉS PIZZINI, Manuel (2021). "La imperiosa necesidad del bacalao: Puerto Rico y Terranova en la EcologíaMundo”, Relaciones Internacionales, n 47, pp. 163-179. 
from the 16th to the 20th century. Spain transferred to the Caribbean colonies its "addiction" to cod, a cheap source of protein for peasants and laborers in the Peninsula, and a foodstuff that became the source of protein for slaves, peasants, and rural workers in the Caribbean.

The British also used that alimentary strategy in their Caribbean colonies, which became importers of salted fish from New England, Labrador, Nova Scotia, and Newfoundland, a territory that dominated the production and processing (culling and salting) of cod.The plantation system required a cheap protein source for the slave labor force that the American codfish market provided. By the end of the 19th century, the supply of New England cod waned (due to a number of reasons), and that opened up an opportunity for Newfoundland to become the most important supplier of fish, a commodity mediated at first through the British authorities, and later by the merchants of the Dominion. The expansion of the productive frontierfrom the 19th to the 20th century-required the investment of capital in larger boats and an increase in effort through the use of nets and long liners in waters distant from the inshore areas.

This paper presents an anthropological and historical analysis of the relationship between Puerto Rico and the producers of salted fish in the North Sea, namely, New England and Newfoundland, through the use of a number of historical and ethnographical studies and, most importantly, through the analysis of primary sources from the General Archive of Puerto Rico, the National Records and Archives Administration in the United States, and the Provincial Archive of Labrador and Newfoundland. This research started, originally, with an interest in understanding the relationship between both markets in their complex export-import relationship, and with a special focus on the 20th century due to the availability of the primary sources. However, this effort led me to the search for other historical and ethnographic sources that documented the social, economic, and political processes that shaped the production of salted fish and the circulation of that commodity in the Atlantic Ocean, with emphasis on the Caribbean.

In that context, the work of Jason Moore proved to be pertinent as a theoretical model to analyze the role of producers and consumers of cheap foodstuffs on a global scale. The World-Ecology model, and the concepts of production frontiers, commodity frontiers, and ecological surplus are extremely useful in understanding how Newfoundland expanded the productive frontier, both horizontally and vertically, by moving the effort from the inshore areas to the offshore banks, which required fishing in deeper waters. In order to provide a cheap commodity to the laborers of the plantation system of the Caribbean, the Newfoundland merchants exploited the unpaid labor of the women and children of the outports (coastal communities) in the processing phase (culling and salting), while forcing the men to look for cash in other subsidiary activities. Low wages and poor working conditions in the curing of fish discouraged laborers to do high-quality work, and therefore, the curing was not always of the required standard for the markets. However, the low-quality fish, the Labrador cure, found its way to the Puerto Rican market.The United States, in the defense of its vested interests in sugarcane cultivation and sugar production, imposed low prices for the importation of salted fish from Newfoundland, and therefore regulated the price per pound at the local markets.

The work of Jason Moore matches the theoretical model developed by Daniel Pauly, a scientist devoted to the study of the global fisheries, and particularly those of the North Sea. For Pauly, the fate of the world fisheries has been in the hands of a threefold expansion (dubbed as the "toxic triad") led by the development in fisheries technologies over the past four hundred years, and accelerated in the 19th and 20th centuries. Those are the geographical expansion, the bathymetric expansion and the taxonomic expansion. That is, global fisheries expanded their productive activities to new geographical frontiers hitherto explored or utilized, including depths. It also expanded the number of species caught, a process that impacted the food chain. Industrialization, and the use of trawler nets in the 20th century, depleted the North Sea fish stocks and destroyed benthic habitats, which contributed to the collapse of 1992.An "undercurrent" flowing in this paper consists of the argument that there is a strong correspondence between the theoretical thinking of Jason Moore and that of the most progressive fishery scientists, exemplified by Pauly.

Over the last thirty years, I have been involved in research and applied work related to fisheries management and the conservation of habitats and fish populations and stocks. In understanding the relationship between Puerto Rico and Newfoundland, I became interested in the history of fisheries management and conservation, and the economic and political forces that contributed to the collapse of the stocks and populations of cod (Gadus morhua) and other Gadidae that forced the 1992 closure and the demise of one of the most important fisheries in our hemisphere. Data and interpretations from a number of studies suggest that, for a long period of time, the 1992 closure reflected the cumulative effect of centuries of the intensification of production, due to the demand for cod from different parts of the world. This paper suggests that Puerto Rico played a critical role in that process, due to the importance of imports from Newfoundland. Throughout the 20th century, Puerto Rico was one of the most important buyers of salted fish, importing as much as all the Caribbean islands at different points in time. That is a line of research that I will continue to pursue, and this paper is a first step in that direction.

Newfoundland tried to maintain the salt fish market of the Caribbean until the 1960s, when Puerto Rico was in the midst of an industrialization and modernization process that reshaped the taste and consumption patterns of the population. By then, cod ceased to be a gastronomic addiction, despite the efforts of two merchant classes, one in St. John's and the other in San Juan.

The article is divided into eight sections that broadly cover the following topics: the history of salted fish consumption and production in the North Sea, Spain, and New England; the British and Newfoundland market in Puerto Rico; cod consumption in Puerto Rico during the 19th century; the relations of production (ecological surplus) that made possible cheap cod;20th century transformations in Puerto Rico, and a final reflection on the role of Puerto Rico and Newfoundland in the World-Ecology.

\section{KEYWORDS}

Newfoundland; Puerto Rico; cod (salted fish); production frontiers; ecological surplus. 
Thtroducción

El desarrollo del capitalismo, según Jason Moore, ha dependido de la expansión de las fronteras productivas (zonas de explotación de mano de obra y recursos) y de la extracción y consumo de insumos baratos. En el Caribe, la producción de caña de azúcar dependió de la importación masiva de africanos esclavizados en plantaciones y de la alimentación de esa fuerza de trabajo con insumos baratos como el pescado salado. Melazas, rones, esclavos y bacalao eran parte de un circuito de mercancías con las que se forjó un segmento de la Ecología-Mundo que continuó desde el siglo XVI hasta la primera mitad del siglo XX. Este artículo explora ese proceso por medio del caso de Puerto Rico y su relación con los centros de producción bacaladera de Nueva Inglaterra y Canadá.

Terranova fue uno de los principales productores y suplidores de ese producto a Puerto Rico y el Caribe, y mantuvo una estrategia de expansión de la frontera productiva, saliendo de la pesca costera para pescar en los grandes bancos mediante un proceso sistemático de acumulación e inversión de capital, articulado por innovaciones en las artes de pesca y las embarcaciones. Este artículo sugiere que el análisis de las poblaciones de peces (traducidos en abastos, recursos a ser explotados) en la larga duración debe tomar en consideración la contribución del consumo por parte de las formaciones sociales ${ }^{2}$. En otras palabras, el consumo de bacalao por parte de Puerto Rico (y otras formaciones sociales) debe ser parte del análisis del colapso de esas poblaciones en 1992. Es una hipótesis de trabajo que estamos elaborando y que aquí dejamos asomar sus primeros atisbos.

Este artículo es el producto de una investigación en antropología histórica sobre la dimensión socio-ecológica de las pesquerías de Puerto Rico, en la que se explora el papel del bacalao en el consumo, el mercado y la alimentación de los puertorriqueños. El estudio de la historia de las comunidades costeras y la producción pesquera nos condujo a examinar críticamente el papel de las importaciones y el consumo de pescado salado en la dieta local. El análisis de contenido de los informes oficiales, de los siglos XIX y XX indicaban una dependencia al pescado importado. Eso nos condujo a investigar la producción y el mercado de bacalao de Terranova a través de trabajos etnográficos, y fuentes secundarias y primarias (documentales) en los archivos de Puerto Rico, Estados Unidos y Terranova ${ }^{3}$.

Nuestra experiencia de trabajo en los aspectos sociales del manejo de las pesquerías nos ha llevado a pensar sobre los procesos de extracción en la larga duración, que pueden ser explicados a través del modelo promovido por Jason Moore, con conceptos como el oikeios, el excedente ecológico y las fronteras de producción (2020). Este tipo de análisis ha sido aplicado

\footnotetext{
Esta investigación fue subvencionada con fondos semilla de la Facultad de Artes y Ciencias de la Universidad de Puerto Rico en Mayagüez. El programa LILLAS-Benson Visiting Resource Professor Program de la Universidad de Texas, en Austin, permitió consultar importantes fuentes bibliográficas. Mi agradecimiento a Carlos Ramos-Scharrón y Megan Raby por esa oportunidad; y a los colegas Marcelo Luzzi, Juan Agar y Eduardo Kicinski por sus comentarios al manuscrito.

2 El concepto de abasto se utiliza aquí para identificar un segmento de la población de peces susceptible a ser pescados, sin soslayar las críticas a dicho concepto que le han hecho Campling y Colas (2021, p. I64).

3 En 1994 explorábamos documentos sobre la pesca en los archivos estadounidenses cuando nos topamos con varios expedientes sobre la importancia del comercio entre Estados Unidos, Puerto Rico y Terranova en el siglo XX y el tejemaneje económico y político que se estableció entre esas formaciones sociales. Eso nos condujo a visitar el archivo de Terranova y Labrador en St. John's y el de la Universidad Memorial en 1997 para iniciar una búsqueda de documentos sobre esos procesos. Posteriormente, y en diversos periodos, visitamos el Archivo General de Puerto Rico para estudiar varios expedientes sobre el tema. Esa información, junto a la extensa bibliografía etnográfica, histórica y económica de la producción de pescado salado a escala global, nos ha permitido abordar ese procesos en la larga duración.
} 
en las pesquerías en la obra reciente de Campling y Colas (202I), obra de la que hemos tomado algunas ideas y sugerencias.

Existe también una interrelación entre los fundamentos de la Ecología-Mundo y los esgrimidos por el especialista en pesquerías Daniel Pauly, quien ha planteado que el colapso de las pesquerías, a escala global, se ha debido a lo que llama "la tríada tóxica": (I) La expansión geográfica: la explotación pesquera a lugares y bancos distantes; (2) la expansión batimétrica: la exploración sistemática y la búsqueda de especies y abastos en aguas más profundas; proceso que requiere de tecnologías más elaboradas; y (3) la expansión taxonómica: la ampliación de las capturas a especies que antes no eran usadas (Pauly, 2019, pp. 2-6). Estos procesos coinciden con lo que Moore ha llamado "la frontera de las mercancías", que se refiere a "la extensión de las relaciones de extracción hacia regiones de poca o mínima mercantilización" y el "movimiento a zonas siempre por explotar" (Moore, 20I3).

Puerto Rico fue, junto a otras colonias y países, un consumidor extraordinario de bacalao para sostener a su población esclava en el siglo XIX y a la enorme masa de trabajadores rurales en el llamado Reino de Azúcar estadounidense en la primera mitad del siglo XX.Aunque el consumo de Puerto Rico fue una fracción del total de bacalao producido por Terranova, la Isla fue uno de sus principales clientes, en ocasiones superando la totalidad de los países del Caribe insular. Fue una formación social que, junto a otras, estimuló una expansión de la frontera de producción pesquera, como ha sucedido también en otras partes del mundo (Campling y Colas 2021, p. 182). La expansión de esas fronteras mercantiles y de producción reconfiguró los hábitats, los ecosistemas y la biodiversidad, por medio de una expansión de la producción y el consumo.

A modo de una hipótesis de trabajo (sobre todo para investigaciones futuras) este artículo arguye que, para entender el colapso de las poblaciones de peces y los abastos, es necesario entender su historia ecológica, los procesos de expansión de las fronteras productivas y la transfiguración de los ecosistemas en función de un incremento en la producción, en la larga duración. Un análisis facilitado por las ideas de Moore y las de Pauly, ideas que han abonado a este primer producto de esa investigación relacionada a la relación entre Terranova y Puerto Rico. Aquí hilvanamos esa historia ecológica explorando en las secciones del trabajo los siguientes temas: el proceso de producción y consumo en Europa; la producción y consumo de bacalao en España, como alimento barato; el desarrollo de esas pesquerías en Nueva Inglaterra y Canadá para abastecer a la fuerza de trabajo esclava de las Antillas; y el desarrollo de un mercado de pescado salado entre Terranova y Puerto Rico en los siglos XIX y XX. Este trabajo concluye que el caso de Puerto Rico y Terranova se adscribe a las dinámicas inherentes en los procesos antes descritos por Pauly y Moore, como veremos en el análisis.

\section{Una historia trasatlántica}

El bacalao y el arenque son dos especies de peces esenciales en la alimentación europea y que fueron el objeto de intensas capturas y distribución mercantil desde la Edad Media. La expansión imperial europea al continente americano, a partir de 1492, significó una extensión de los intensos procesos de captura de peces en la región oriental del Mar del Norte que llevó inclusive a la 
merma de esos abastos (Fagan, 2006; Bolster, 20I2). Es por ello que el desarrollo y la expansión de esa economía-mundo tuvo un interés muy acusado en la explotación de los recursos pesqueros que eran vistos por exploradores y colonos como inmensos, posiblemente en comparación con el declive observado en las capturas europeas. Bolster y Fagan, quienes han estudiado este asunto a fondo han enfatizado que, para Nueva Inglaterra y Terranova, el proceso de ocupación de esos territorios, la expansión colonial y las posibilidades de asentamientos giraron en torno a la relativa abundancia de los abastos pesqueros, pero sobre todo del bacalao.

Las empresas del West Country establecieron campamentos pesqueros (llamados plantaciones) en las costas de Terranova para capturar bacalao y proveerlo a Inglaterra, Portugal y España, estos últimos grandes consumidores de bacalao (Pope, 2004) ${ }^{4}$. Por muchos años esa fue una pesca dominada por el West Country, con pescadores migrantes estacionales quienes operaban por temporadas en las costas de Terranova, pescando, salando y embarcando el bacalao a Inglaterra y España, a los centros importadores más importantes: Bilbao y Sevilla (Graffe, 20I2). España también se unió a la empresa pesquera de las aguas de Terranova enviando a sus flotas gallegas y vascas a pescar a esas aguas, asunto al que se dedicaron con ahínco hasta el siglo XVIII (Ménard 2008).

España desarrolló un extraordinario gusto gastronómico por el bacalao, "una adicción" que se convirtió en un plato esencial, por ser una proteína barata, en las mesas de las familias pobres (Graffe 2012:56). Fue el resultado de una transición alimentaria a partir de 1640, cuando los españoles fueron dirigiendo su gusto y necesidad de los clupeidos (sardinas, boquerones y arenques) hacia los gádidos (Cubillo de la Puente, 1998, p. 138). A partir de 1612 España satisfizo la demanda por bacalao comprándoselo a los británicos por la vía de los mercaderes del West Country quienes lo intercambiaban por oro, plata y lana de las ovejas merino para suplir la industria de los paños españoles. España fue de las últimas naciones imperiales en reclamar derechos de pesca en Terranova, y quedó prácticamente fuera de esos bancos pesqueros por medio del Tratado de Utrecht, lo que hizo en extremo difícil abastecerse de bacalao. Con ese tratado, Gran Bretaña mantuvo el control sobre Terranova, aunque los ciudadanos de Estados Unidos mantuvieron derechos de pesca sobre algunas costas (hasta 1910) y Francia tuvo control sobre los bancos pesqueros de sus islas St. Pierre y Miquelón (Neary, 1988, p. 4).

Desde el siglo XVI existía en España una red de comercialización y distribución de pescado por todo el territorio peninsular, a través de arrieros, maragatos y regatones, quienes transportaban y trajinaban el pescado desde los centros de producción e importación a las localidades de consumo. Gallegos y vascos desempeñaron un papel crítico en la distribución del bacalao, por haberlo pescado o por tener relaciones mercantiles con el West Country y tener, inclusive, agentes compradores en Londres (Cubillo de la Puente, 1998).

En ese devenir hay tres datos que ameritan resaltarse: (I) a España llegaba el bacalao verde (light cure) y el marchante, que era un bacalao de alta calidad; (2) el bacalao de baja calidad era vendido a las Antillas británicas; y (3) España mantuvo la importación de bacalao de diversa procedencia, pero dominando el de Terranova, a través de mercaderes ingleses, sobre todo en

\footnotetext{
4 West Country es el nombre de la región del suroeste de Inglaterra, compuesta por los condados de Dorset, Devon, Cornwall y Somerset,
} que formaron el corazón de las empresas bacaladeras que ocuparon a Terranova. 
los siglos XIX y XX (Ryan, 1980). Esto último arroja luz sobre el papel de Puerto Rico en ese "inmenso comercio". Durante los primeros siglos de la ocupación española el bacalao que llegaba de la Península era muy escaso, lo que provocó que la Isla iniciara un comercio legal e ilegal para abastecerse del bacalao angloamericano (Ortiz-Cuadra, 2006, p. 147).

\section{Los Estados Unidos: un imperio en el porvenir de Puerto Rico ${ }^{5}$}

La pesca fue una actividad de enorme importancia económica, social y política en Nueva Inglaterra, sobre todo en Massachusetts y Maine. La riqueza de los bancos pesqueros, costas y estuarios de esa región proveyeron una enorme variedad y cantidad de especies de peces que fueron el objeto de una explotación sostenida por muchos años, entre ellas el arenque, la macarela, el arenque americano y el bacalao. La actividad pesquera generó la forja de capitales por medio de la construcción y mantenimiento de embarcaciones, la producción de manufacturas usadas en la actividad, la venta de vituallas, la producción de sogas y maderas y el sector de los seguros para paliar los riesgos de la empresa pesquera. La pesca fue dirigida por unas clases mercantiles que se tornaron en poderosas elites de gran influencia política. El sector pesquero reclutó a una gran cantidad de hombres en esa región, quienes combinaban labores agrícolas con la pesca por temporadas (Magra, 2009).

La pesca del bacalao en Nueva Inglaterra encontraba salida comercial en las Antillas donde era intercambiada por varios productos, pero sobre todo por melaza usada para la fabricación de ron. Los dueños de las plantaciones en las Antillas francesas, británicas y españolas compraban el bacalao para alimentar a la población esclava que era la fuerza de trabajo esencial para la producción de caña de azúcar. Una parte de la producción de bacalao era vendida en Europa, sobre todo el bacalao marchante de la más alta calidad, mientras que el de baja calidad (refusegrade cod) era llevado a las Antillas, preferiblemente francesas cuyos plantadores ofrecían la melaza a un precio más bajo (Magra, 2009, p. 132).

Para Magra, la inversión de capital, de esfuerzo y de trabajo en la producción de bacalao y el comercio con las Antillas fue de tal magnitud e importancia que todos esos actores sociales se vieron obligados a enfrentarse al gobierno británico cuando este comenzó a establecer restricciones al mismo. Las presiones políticas en las cámaras de los lores y de los comunes — por parte de los representantes de los mercaderes delWest Country y de los dueños de plantaciones en las Antillas - fue tal que empujaron legislación para imponer aranceles sobre la melaza importada a Nueva Inglaterra. El gobierno británico prohibió el comercio con las Antillas (en defensa de los plantadores absentistas), el avituallamiento de las "plantaciones" de Terranova, por parte de Nueva Inglaterra, así como la pesca de esas firmas en las aguas de Terranova y Labrador. Esa convergencia de factores empujó al sector marítimo de Nueva Inglaterra a desarrollar una marina de guerra para enfrentarse a los británicos, acción que tuvo como efecto la independencia de esos territorios y mayor poder para esas clases comerciales y plantadoras, muchas de las cuales tenían vínculos económicos con la pesca de bacalao.

En 1898 Estados Unidos, a raíz de la Guerra Hispanoamericana y el Tratado de París, tomó posesión política de la Isla e instituyó un gobierno militar, producto de su invasión. 
Uno de los estados con mayor prominencia en el sector pesquero lo fue Maine, uno de los más pobres. En 1830 Maine había consolidado una población de descendientes de europeos que se dedicaron a la pesca. Una combinación de productores independientes e inversiones por parte del capital mercantil provocó la forja de firmas pesqueras que operaban balandros y goletas en los bancos cercanos a la costa (O'Leary, I 98I, p. 30). Una embarcación podía tener varios dueños que invertían su capital, proveniente del comercio, la construcción de navíos y la agricultura. Además del bacalao, los pescadores de Maine invirtieron mucho esfuerzo en las capturas de la macarela (Scomber scombrus), un pescado por el que se conseguían buenos precios.

Los pescadores y las clases mercantiles de ambos estados aprovecharon que en ese período la corona británica reabrió (formalmente, pues el contrabando siempre fue una opción) los puertos de las Antillas al comercio con Estados Unidos, un mercado que había sido importante desde el siglo XVII. La ola migratoria europea de la primera mitad del siglo XIX contribuyó a ampliar el mercado de consumidores de pescado en Nueva Inglaterra, compelidos por las regulaciones religiosas católicas y anglicanas. El bacalao y las macarelas eran productos esenciales en la mesa de esos nuevos migrantes, como lo eran también para la fuerza de trabajo esclava en las Antillas.

Es importante afirmar que la historia del bacalao, desde el siglo XVIII hasta nuestros días, es también la historia de la intervención estatal en su desarrollo. Ese ha sido el caso de Terranova (Wright, 200I) y el de Maine, estado que instituyó a finales del siglo XVIII el sistema del bounty, un subsidio para aquellas personas y firmas que pescaran para exportar a Europa y al Caribe. Este subsidio contribuyó a incrementar la inversión de capital en la renovación de los medios de producción, lo que ayudó a incrementar la composición orgánica del capital en las pesquerías, por medio de embarcaciones y operaciones de mayor tamaño (O'Leary, I 98I, p. 68).

Este es un momento importante para el que podemos aplicar los postulados de la tríada tóxica de Pauly (2019) y de la "extensión de las relaciones de extracción" que plantea Moore (20I3). Las firmas de Maine empezaron a pescar de manera agresiva en las aguas de Terranova y Labrador, además del Golfo de San Lorenzo, la Bahía de Fundy y Nueva Escocia; sin duda, una amplificación de las operaciones pesqueras en aguas más distantes y en zonas variadas. El bacalao pequeño de Labrador era vendido en Boston y de ahí era exportado a Bilbao bajo el rubro de "Bilbao fish" (O’Leary, I 98I, p. 167). En 1832 la mayoría de las exportaciones de pescado de Maine (90\%) iban rumbo a las Antillas, principalmente a Cuba y Haití, seguido de Puerto Rico, Santo Domingo y los enclaves azucareros británicos en último lugar. Ese mercado continuó durante toda la primera mitad del siglo XIX debido a la imperiosa necesidad de los hacendados y plantadores de tener una fuente de proteína animal barata que pudiese transportarse sin dañarse y el bacalao cumplía esos requisitos (O’Leary, I981, p. I7I).

\section{La circulación de mercancías y la centralidad del bacalao en el siglo XIX}

Las políticas liberales españolas del siglo XIX promovieron en Puerto Rico el desarrollo de sistemas de producción agrícolas para el mercado exterior.A tal efecto, la Real Cédula de Gracias de 1815 estimuló la migración de extranjeros católicos y con ellos sus capitales, para invertirlos 
en el comercio, la caña de azúcar y el café. El café y el azúcar requirieron la importación de mano de obra esclava, que se insertó en los campos de cultivo, en el procesamiento de esos cultivos, en el mantenimiento de las bestias y los campos y en la navegación. De España llegaba el bacalao, pero ese incremento en una fuerza de trabajo que debían mantener al más bajo costo, empujó a estancieros y hacendados a importar bacalao de Nueva Inglaterra y Terranova, con la anuencia del gobierno británico y las clases mercantiles del West Country y de St. John's, que ya empezaban a convertirse en unos poderosos grupos comerciales. El gobierno español en la Isla había ordenado que la fuerza de trabajo esclava estuviese bien alimentada. La directriz, lista de alimentos y raciones incluía ocho onzas de bacalao o de macarelas diarias (Figueroa, 2005, p. 92). Independientemente de la manera en la que los hacendados siguieron esa directriz, el dato es que ambos pescados eran provistos por las flotas pesqueras de Nueva Inglaterra, Terranova y Labrador.

El trabajo de Cruz M. Ortiz Cuadra provee unos datos esenciales para entender el papel del bacalao en la dieta y en las importaciones desde el siglo XIX, que coinciden con el auge de la población esclava y la militar. Coincide también con un giro importante de las relaciones comerciales entre España e Inglaterra, en relación a esta colonia, que se encontraba en un auge de la economía agrícola de exportaciones, con el café y el azúcar, lo que consolidó una poderosa clase dominante formada por dos sectores encontrados: los hacendados y los comerciantes, ambos con dinero para adquirir bacalao de buena calidad para su mesa y bacalao de tercera para su fuerza de trabajo.

Gran Bretaña se movió agresivamente, en el siglo XIX, en el mercado puertorriqueño con manufacturas, metales, carbón, maquinaria para la producción agrícola, maderas y alimentos como el arroz, las harinas y el bacalao. Según se desprende de la correspondencia consular, las mieles de Puerto Rico eran preciadas para la producción de rones, mercancía que intercambiaban por el bacalao “canadiense” que era preferido a los de Nueva Inglaterra (Dávila-Cox, 1996, p. 96).

La historiadora Emma A. Dávila-Cox presenta un incremento en las importaciones de bacalao y merluza desde 1849 donde era cerca de 8 millones de libras hasta llegar a casi 20 millones en 1879 , con una tendencia al descenso hasta 1889. A partir de ese momento se dispararon en alza las importaciones hasta llegar a cerca de un poco más de 45 millones de libras en 1897 , que llegaban en su mayoría de Gran Bretaña (22.092.000 libras), seguido de Estados Unidos con poco más de 2 millones de libras (p. 152).A pesar de que la mayoría del bacalao venía de Canadá, también llegaba de Gran Bretaña pescado en el Mar del Norte europeo, todos con aranceles “modestos" (Dávila-Cox, 1996, p. 235)'.

Las innovaciones tecnológicas y la presión pesquera sobre el bacalao, la macarela y el arenque americano (menhaden) a mediados del siglo XIX causaron una reducción en esas poblaciones en Terranova, Nueva Escocia y Nueva Inglaterra. El uso del palangre (el tub-trawl, una línea con cientos de anzuelos) reemplazó en importancia al cordel de mano en la pesca costera y en los bancos de Terranova, incrementando en varios órdenes de magnitud las capturas cuyo volumen de capturas se había reducido debido a la presión pesquera existente y a los cambios dramáticos en las temperaturas de esas aguas (Bolster 2012, Pp. I22-I24). Esos dos procesos, uno natural

6 Ortiz Cuadra indica que el consumo en 1897 se estimaba en unas 55 libras anuales por persona (p. I50). La gráfica que presenta puede exagerar las cantidades importadas pero la información que recoge de la Balanza Mercantil lo sitúa en 45.3 I I.535 millones de libras en I897. 
(descenso en las temperaturas y reducción en las poblaciones) y otro humano (incremento en el esfuerzo pesquero y las innovaciones) coincidieron para que las firmas pesqueras de esa región mantuvieran la presión sobre esa trama trófica, en momentos en los que el Caribe mantenía una alta demanda por esos alimentos. Ese esfuerzo se movió agresivamente hacia los grandes bancos (la pesca "de afuera", offshore) a zonas distantes de la costa, que requirió la inversión de capital en embarcaciones de mayor calado y capacidad, para viajes de mayor duración (2012, pp. I35-136). Ese siglo también fue testigo del uso de las redes de ahorque (una técnica europea) en la pesca costera, que también tuvo su impacto sobre las poblaciones de peces.

A partir de 1900 las aguas de Terranova y Labrador experimentaron temperaturas más altas que se reflejaron en un aumento en las poblaciones de peces. No obstante, 400 años de capturas habían surtido su efecto y las firmas pesqueras continuaron innovando, introduciendo las redes de fondo (beam trawls y otter trawls), las redes arrastreras, arrastradas por grandes barcos de vela y luego por vapores. Ese proceso aceleró la disminución de los abastos, tan importantes para suplir a la fuerza de trabajo caribeña en la primera mitad del siglo $\mathrm{XX}$.

Ortiz Cuadra señala que, en comparación con I897, las importaciones de pescado salado y otras conservas se redujeron durante la primera década de la ocupación estadounidense en la Isla, debido a la protección que ejerció Terranova y Labrador sobre sus bancos pesqueros, vis-àvis las incursiones de las flotas de Nueva Inglaterra (p. I56). No obstante, la pesca y producción de bacalao de Nueva Inglaterra venía de capa caída en el siglo XX. Nueva Inglaterra se movía entonces aceleradamente a la producción de pescado para venderlo refrigerado, lo que no era del interés del mercado de Puerto Rico.

\section{El bacalao en la transición del siglo $X \mid X$ al $X X$}

"El negocio del azúcar es para Puerto Rico, lo que el bacalao es para Terranova"”. Esta sentencia resume la relación entre ambos territorios coloniales: un vínculo basado en la importancia superlativa que adquirió la producción azucarera con las corporaciones estadounidenses a partir de 1898 y la imperiosa necesidad de proveer a la fuerza de trabajo una proteína a bajo costo. Tan temprano como 1899, el gobierno estadounidense clamaba por la entrada de bacalao libre de aranceles desde las provincias británicas, asunto que se discutió durante toda la década. En ese año fiscal (1898-1899) la inclinación de la balanza comercial era abrumadora a favor del pescado salado de Canadá y Terranova. La transición de poderes imperiales significó un cambio en las cantidades de bacalao importado de Estados Unidos, en comparación con el de las provincias norteamericanas dominadas por Inglaterra, como Nueva Escocia, Labrador y Terranova. A partir de 1900, Puerto Rico comenzó a importar el pescado salado canadiense - libre de aranceles hasta 1910 - en grandes cantidades para sostener a esa fuerza de trabajo. El año fiscal 1909-1910 fue definitorio en esa transición ${ }^{8}$. Todas las fuentes consultadas apuntan a que esa primera mitad

\footnotetext{
Provincial Archives of Newfoundland and Labrador and (PANL), GN-38, 1939. The Report of Mr. George Lewis on the Puerto Rico Salt Codfish position.

8 Durante la primera década del siglo $\mathrm{XX}$ hubo bastante variación —en términos monetarios-entre el pescado (bacalao, arenques y sierras) importado de Estados Unidos y el traído de naciones extranjeras, pero la tendencia observada apunta a una inclinación hacia el pescado importado de naciones extranjeras. De 1901 a 1910, la balanza se inclinó hacia Estados Unidos en 1906 (por muy poco) y 1908 . National Archives and Records of the Administration (NARA). C 884, Box 43. Summary of transactions in the US Custom District of Porto Rico for
} 
del siglo XX significó una ampliación de las actividades pesqueras en Terranova para sostener al mercado caribeño, del que Puerto Rico era uno de los actores principales, y que significó una intensificación en las actividades extractivas y una expansión hacia diversos bancos de pesca.

La lista de compradores de bacalao de Terranova estaba conformada por una matriz de consumidores atados en un segmento muy particular de la Economía-Mundo de ese momento, cada uno construyéndose como una variable crítica en la trama trófica y comercial de esa EcologíaMundo, que provocaba la implementación de la tríada tóxica de Daniel Pauly y de la expansión de las fronteras extractivas que sugiere Jason Moore.Todos esos procesos estaban atados a diferentes economías y sistemas de producción, vinculados a enclaves agrícolas, coloniales y poscoloniales, muchos de ellos cañeros en el Caribe y en Latinoamérica.

\section{El bacalao y las relaciones de producción que lo hicieron posible}

A ambos extremos del Atlántico, ese proceso de extracción fue dominado por el capital mercantil, representado por los merchants de Terranova y por las casas comerciales de origen español ubicadas en Puerto Rico, quienes controlaban el suplido del bacalao. El siglo XIX representó en Terranova la forja y consolidación de la sociedad de los outports, es decir, las localidades y comunidades pesqueras permanentes, formadas con migrantes - convertidos en residentes - del West Country y de Irlanda (Ryan, 1980). La sociedad del outport estaba caracterizada por la pesca costera (inshore), la importancia de la familia, los comerciantes y las villas o comunidades costeras (Sider, 1986, p. 2I). Esos miles de residentes consolidaron el proceso de pesca y cura del bacalao en sus costas, invirtiendo en sus embarcaciones y la infraestructura portuaria. No obstante, el proceso de producción, de avituallar las operaciones y las unidades domésticas dependieron del capital de los comerciantes, quienes subvencionaban esa operación para luego remunerarlos a precios que eran descubiertos en el momento de la paga, lo que ponía a las familias de los outports en desventaja con los comerciantes quienes a su vez le vendían las provisiones y vituallas con artículos tomados a crédito en sus tiendas. Este sistema de intercambio entre comerciantes y pescadores se conoció como el sistema del truck, un trueque mercantil (Sider, 1986). En el siglo $\mathrm{XX}$ la sociedad outport proveyó los mecanismos para que se pudiera producir el bacalao a bajo costo y pudiese ser comprado por las familias proletarias del sector cañero en Puerto Rico y en otros países. En cierta medida, ese proceso corresponde con lo que Moore ha llamado el excedente ecológico, un proceso mediante el cual el capital se apropia del trabajo no-remunerado de sectores de la población al mismo tiempo que expande la apropiación (el expolio) de la naturaleza, expandiendo la frontera geográfica a zonas más distantes o a las profundidades de la tierra y del océano (Moore, 2020, pp. I 17- I20; Campling y Colas, 2021, p. 167).

En una apretada síntesis, podemos indicar los siguientes procesos que facilitaron esa expansión en el sistema de extracción: (I) El sistema del truck, un trueque de pescados por artículos de consumo; (2) Esa relación — desprovista del uso de moneda - era lo que mantenía a las unidades domésticas en un sistema de producción dominado por los comerciantes, en el ámbito de la circulación de mercancías; (3) Ese sistema de obligaciones y deudas coartaba la inversión de capital por parte de los pescadores, como ocurrió en ciertas partes de Nueva

the fiscal year ending June $30,1909-1910$. 
Inglaterra (Sider, I 986, pp. 22-29); (4) El trabajo de las unidades domésticas funcionaba con el uso de la fuerza de trabajo no-remunerada de los niños y las mujeres en el proceso de limpieza y cura de los pescados, tal vez la principal tarea relacionada con el potencial comercio de las capturas (véase a Antler, 1982, p. 5; Moore, 2020, p. 74). Ante la ausencia de dinero en efectivo, los hombres invertían su tiempo fuera de la temporada de pesca en algunas actividades extractivas que les proveían una remuneración en efectivo, siendo la más popular y peligrosa la cacería de focas (Ryan, 1980, pp. 45-46).

El asunto de la explotación de la fuerza de trabajo y la precariedad de la vida de las familias dedicadas a la pesca en Terranova era una preocupación de la oficialidad. En 1935 el Departamento de Recursos Naturales señalaba el problema de la deuda en los outports, era identificado como un mal (evil) que sometía a los pescadores a una relación desigual con los comerciantes, al estar atados a los precios de las mercancías y las vituallas, de las que dependían. Este era un problema que afectaba a cerca de 30.000 familias a las que se les hacía muy difícil producir para los mercados internacionales, mientras pasaban serias dificultades para sostener sus familias con los precios impuestos por los comerciantes?.

Los precios bajos para el bacalao en los mercados internacionales, y los altos precios de las vituallas y provisiones en esa relación de crédito y endeudamiento, sometían a los productores "independientes" a condiciones deplorables, "de miseria”. Los comerciantes se quejaban de que las familias de los outports carecían de honestidad y disciplina para el trabajo, lo que se reflejaba en la pobre calidad de la cura y el escogido de grados de bacalao (culling), lo que les traía problemas a los comerciantes en los mercados internacionales ${ }^{10}$. Lo que salvaba la situación era que esas familias "disfrutaban de las ventajas" de la vida campesina, es decir, podían ocupar las tierras libremente, producir para su subsistencia (cabras, ovejas, papas, repollos y otros), lo que "explica su continua participación en la pesquería". Aunque ese informe no lo menciona, en diversos momentos de la historia del siglo XX, a esas familias el gobierno del Dominio les proveyó una asistencia financiera (the Dole) para ayudarles a sobrevivir y para sostener al capital mercantil de Terranova.

\section{Siglo XX, cambalache: capital mercantil, trabajo y precios del bacalao}

En el siglo $\mathrm{XX}$ el bacalao no era la proteína obligada para los militares, pero sí para las familias trabajadoras en el sector azucarero que constituyó el corazón de las inversiones capitalistas estadounidenses en la primera mitad del siglo XX. Ortiz Cuadra sugiere, según los datos que examinó, que hubo una difícil contracción en la oferta y en el consumo de bacalao por parte de los puertorriqueños, y que de 1929 — la Gran Depresión- a 1935 la cantidad de todos los alimentos disponibles se redujo (2006, p. I57).

Los datos de las importaciones apuntan a una merma en las importaciones de bacalao,

\footnotetext{
PANL GN, General Administration S 2-I-I4b. Report of the Department of Natural Resources (sin fecha).

10 PANL GN, Miscellaneous. S 2-I-I4b. "A menos que aumenten los precios mundiales del pescado, no hay perspectivas de que una minoría importante de pescadores sea abastecida para la pesca en el marco del actual sistema de crédito mercantil". Traducción propia. Confidential Interim Report to the Commission of Government by J. H. Gorvin, 6, October, 1938.
} 
pero Puerto Rico era, en esos momentos, un importante consumidor del bacalao producido en Nueva Escocia, Labrador y Terranova, a pesar de que el bacalao se vendía a precios muy bajos. Puerto Rico, antes de 1923, importaba cerca de 3.000 toneladas métricas por año, con un pico de 6.588 toneladas métricas en 1922. De 1933 en adelante, las importaciones disminuyeron hasta oscilar entre 1.000 y 3.000 toneladas. Pero para Terranova eso era un tremendo negocio, puesto que "la posición de Canadá se mantuvo mejor en los países del Caribe que en los de Europa o Sudamérica” (Gerhardsen, 1949, p. 30).

Los datos sugieren que Puerto Rico importó y consumió, en la primera mitad del siglo $\mathrm{XX}$, menos bacalao de Terranova y otras jurisdicciones que durante el período álgido del siglo XIX. No obstante, hay tres procesos que son necesarios entender: (I) Terranova vendió más pescado salado a diversos países de todo el Atlántico, por lo que Puerto Rico era un mercado entre varios; (2) Pero no era un mercado más, era uno en extremo importante, a pesar de tener precios límites y control de precios por parte del gobierno estadounidense, sobre todo durante la Segunda Guerra Mundial"'; (3) Curiosamente, la multiplicidad de procesos sociales y económicos que mantenían a la fuerza de trabajo en condiciones paupérrimas en Terranova, orientaban la producción hacia un bacalao barato (la cura conocida como Labrador), con muy poca atención a la cura y selección, lo cual tenía su venta asegurada en los mercados caribeños y, claro está, en Puerto Rico. Esa diferencia entre el costo de producción (asumido por las unidades domésticas de los outports) y las ganancias recibidas por los comerciantes, hicieron del mercado de Puerto Rico uno ganancioso para la clase mercantil de Terranova (Antler, 1982).

Los precios en la compraventa beneficiaban a la clase mercantil de Terranova y la de los importadores de Puerto Rico. Dentro del muy estrecho margen de negociación, ambas partes luchaban por cada centavo en las transacciones, en una relación trasatlántica en la que las familias pescadoras vivían en precario, para producir una proteína para los platos de las familias obreras de la caña en la Isla.Alrededor de 1930 tanto las familias pescadoras como las obreras vivían en la miseria, produciendo para clases capitalistas en la producción y circulación de mercancías, y ambas familias estaban inmersas en unas relaciones desmonetizadas, remuneradas por unos vales a ser redimidos en las tiendas de raya de las centrales azucareras y en las de los merchants. Ese sistema estaba hilvanado por el proceso de circulación de mercancías, enhebrado desde St. John's y San Juan por los sectores comerciales; unos trazaban su linaje a las familias del West Country y los otros a distintas regiones de España.

Ese proceso de compraventa del bacalao estaba plagado de conflictos, quejas por los embarques y la calidad del bacalao y los arenques, y por el enrevesado sistema de envíos y transacciones. En Terranova, las familias pescadoras tuvieron grandes esfuerzos para organizarse y conseguir mejores precios, o para que los comerciantes pactaran de antemano el precio de compra del pescado, pero eso se convirtió en un peje muy elusivo. Quienes se organizaron bien -en ocasiones con un poco de presión gubernamental- fueron los comerciantes. En 1932 el gobierno los forzó a armar la Junta de Bacalao Salado (Salt Codfish Board), que luego se transmutó, en 1936, en el Newfoundland Fisheries Board (NFB). Esta organización se formó para resolver

\footnotetext{
"El gobierno estadounidense desarrolló el Programa de Emergencia Caribeña para asegurar la oferta de alimentos en Puerto Rico y las Islas Vírgenes Estadounidenses y controlar los precisos. National Records Archives and Records of the Administration. Box 24, Office of the Territories. Varios documentos.
} 
algunos conflictos de interés que tenían los miembros de la junta anterior y con los objetivos de organizar, concentrar y consolidar el proceso de producción (la calidad del producto) y la venta en los mercados internacionales (Antler, 1982, pp. 235-237). La NFB organizó a grupos de exportadores, según los destinos de las mercancías y para ello formó el Puerto Rico Exporters Group.

El gobierno de Terranova interesaba racionalizar el proceso de producción y había recomendado, a través de varias comisiones, que se debía estimular la producción de un pescado de mayor calidad, para poder tener el beneficio de aquellos mercados dispuestos a pagar precios más altos. La clase comerciante, al parecer, se resistió a ello ya que para estos "el margen de ganancia más amplio no estaba en el pescado de alta calidad, sino en aquellos de la más baja calidad" y su salida a mercados como el de Puerto Rico (Antler, 1982, p. 239).

Por esa razón, los comerciantes casi se desentendieron del procesamiento, lo dejaron en las manos de las familias pescadoras y a ellos les achacaban todos los problemas, mientras extraían ganancias de la cura de baja calidad. Es en ese contexto que el mercado caribeño se expandió en las primeras décadas del siglo XX. La evolución de estos grupos continuó con la forja de un cartel de exportación (considerado como "malévolo") cuya preocupación consistía en vender ese bacalao de baja calidad:The Newfoundland Association of Fish Exporters, Ltd. (NAFEL), compuesto por los exportadores más poderosos que podían pagar la cuota de \$10.000 (I 982, pp. 244-245).

Estas organizaciones de producción y mercadeo trabajaron intensamente con el sector comercial de Puerto Rico, para asegurar la venta del bacalao, contra viento y marea; es decir, a pesar de los bajos precios, el control de precios de Washington D.C. durante la guerra y las jugarretas y quejas de los comerciantes de Puerto Rico. Hubo momentos (de 1938 a 1940, por ejemplo) en los que el negocio del bacalao se hizo casi sin ganancias y tuvo el gobierno de Terranova que proveer asistencia a las familias pescadoras para que pudieran sobrevivir ${ }^{12}$. Terranova dependía, a pesar de los bajos precios, del mercado puertorriqueño para sobrevivir como una economía basada en el pescado salado ${ }^{13}$.

NAFEL fue, según la apreciación de David Alexander (1977), exitosa en su misión de organizar a los exportadores en Terranova según los mercados a los que servían, y organizar a los compradores y adjudicarles cuotas como lo hicieron en Puerto Rico. La documentación examinada permite apreciar las diversas maneras en las que los comerciantes en Water Street, St. John's, coordinaban el difícil mercado boricua, donde el sector comercial de origen español mantenía su presión en torno a la calidad de los embarques, los precios, el uso del dólar estadounidense frente a la devaluada moneda canadiense, sobre todo cuando era un mercado que favorecía a los compradores (Alexander, 1977, Pp. II6-II7). Terranova venía perdiendo terreno en los mercados europeos, asunto que agravó la guerra. Otros países, como Islandia, Noruega y Francia se movieron agresivamente con pescado de mejor calidad. Esto provocó que se incrementaran las exportaciones al Caribe (Gerhardsen, 1949, p. 36). Puerto Rico era, según Alexander, "el más

\footnotetext{
12 PANL. GNI-34, Fisheries General Correspondence. Carta del gobernador de Terranova al embajador británico. 24 de agosto de I942. Los subsidios, además de dinero, consistieron en precios bajos para la sal y la gasolina de las embarcaciones.

13 PANL. GN 38. NAFEL, Memorando al Comisionado de Recursos Naturales. 1939. "En nuestra opinión, Portugal, Brasil y Puerto Rico son considerados mercados claves. Puerto Rico en un mercado bastante grande... que compra lo más barato posible, lo que derrota nuestro interés por un mercado más ganancioso. Pero dependemos de este mercado para la venta de más del $50 \%$ de nuestra producción normal de Labrador".Traducción propia.
} 
grande y el más sensitivo de los mercados caribeños" (1977, p. 256) ${ }^{14}$ y el hecho de haberse movido, durante la guerra, a consumir el pescado de la clase Labrador, fue una bendición para NAFEL, pero luego de la guerra, con los precios levantados, entonces NAFEL ejerció más presión sobre los importadores para comprar otras clases de pescado ${ }^{15}$. No obstante, ya era muy tarde, como veremos a continuación.

\section{Las transformaciones pesqueras y gustativas en Terranova y Puerto Rico a partir de 1950}

La fecha de 1950 es un hito temporal en tres importantes procesos: (I) la "modernización" y expansión de la flota de barcos arrastreros de Terranova a bancos y pesqueros más distantes; (2) el incremento en la competencia pesquera por el bacalao en los caladeros de Norteamérica por flotas españolas, francesas, soviéticas, cubanas y noruegas, entre otras; y (3) el proceso de modernización de Puerto Rico que tuvo como efecto un cambio en la dieta boricua, en la que se fue abandonando el bacalao como pieza central.

Cada uno de estos procesos amerita un artículo por separado, pues esa tríada de procesos configuró el escenario de la salida de Puerto Rico del mercado del pescado salado,y el incremento en la producción pesquera que desembocó en el colapso de la pesquería y los outports en 1992. No obstante, presentamos aquí un resumen de los mismos:

I. El gobierno y el sector privado de Terranova estaba conteste en que el territorio debía moverse agresivamente hacia mercados diferentes al del pescado salado, como el del pescado congelado. Ese producto y sus derivados (los pedazos de pescado empanados, fish sticks) tenían su nicho en las mesas estadounidenses y Terranova apostó a ello con el objetivo de obtener mejores precios, salir del anticuado mercado de la salazón e incrementar la inversión de dinero y capital en las pesquerías. El gobierno invirtió mucho dinero y esfuerzo en esta empresa, con el fin de sacar a Terranova del atraso, hacer desaparecer a las familias pescadoras de los outports, para crear una clase de trabajadores asalariados industriales en los barcos arrastreros, los barcos congeladores y las plantas procesadoras. En esa visión de mundo de la modernización, los obreros industriales y asalariados eran un paso superior a la clase pescadora, parcialmente integrada a un capitalismo de corte mercantil que predominó hasta entonces (Wright, 200I). Mientras eso sucedía, las aguas del Mar del Norte en este hemisferio se colmaban de arrastreros que laceraban la capacidad reproductiva de los peces de fondo y lentamente alteraban y estropeaban los hábitats bentónicos con su arrastre del fondo (Pauly y MacLean, 2003). Los eventos de 1992 confirman que se produjo un agotamiento de la naturaleza, reflejado en el colapso de los abastos (Moore, 2020).

2. En esa aparente bonanza, provocada por la expansión de las fronteras horizontales y

4 Traducción propia.

15 El informe de las condiciones del mercado, para enfrentar el período de la posguerra describía la situación de la siguiente manera: "En términos generales, la demanda en Puerto Rico se rige en gran medida por el precio. Los consumidores en su mayoría se encuentran entre las clases trabajadoras cuyo poder adquisitivo es limitado. Esto se demuestra en los tipos de pescado que se capturan en el mercado, que son pescados pequeños, de orilla seca y curada blanda y labrador semiseco. Esos tipos pertenecen a la clase de pescado de menor precio producido en Terranova". (The Newfoundland Fisheries Board, 1946, p. 46). Traducción propia. En ese momento, el bacalao Labrador había perdido terreno en los mercados del Mediterráneo. 
verticales, participaron las flotas de diversos países que ya he mencionado. Ese incremento en el esfuerzo pesquero debilitó los abastos y redujo la capacidad de recuperación de las poblaciones de gádidos en toda la región. Terranova y Canadá lucharon a brazo partido por expandir la jurisdicción pesquera a 12 millas y luego a las 200 millas, para poder expulsar a sus competidores y aprovecharse de ese recurso a plenitud. No fue fácil pues Estados Unidos presionó para que no se lograra y el gobierno canadiense trabajó el asunto con extremo cuidado para no lacerar las relaciones económicas y políticas con ese país. Eso no impidió que diversas flotas invadieran sus aguas para pescar y hasta provocaran conflictos cercanos a la violencia, como lo fue el caso de "la guerra del fletán" con un barco español en 1995 (Harris, 1998, pp. 23-30).

3. Los puertorriqueños comenzaron a abandonar la dieta de bacalao y verduras, al insertarse en el mundo industrial creado por el proyecto de modernización del país conocido como "Manos a la Obra" (Ortiz-Cuadra, 2006). Con un mayor ingreso per cápita (y poder adquisitivo), producto del trabajo en la manufactura y los servicios, los consumidores se movieron hacia otros alimentos como el pollo congelado, la carne de res, los mariscos y pescados congelados y los productos procesados y enlatados. Ese proceso coincidió con un descenso en la producción de verduras (que acompañaban al bacalao en el plato) y un incremento en los supermercados, que intentaban borrar para siempre la imagen retardataria de pequeños colmados y puestos en las plazas de mercado de los pueblos con los productos expuestos al aire. Los supermercados ofrecían una experiencia de consumo diferente, con aire acondicionado y productos empacados. En ese trajinar, el bacalao dejó de ser pertinente y de 1950 a 1970 el consumo per cápita se redujo de II,9 libras a 5,42 libras (Ortiz Cuadra, 2006, pp. 163-164).

Ante ese cuadro y desde 1950, los exportadores de Terranova trabajaron incansablemente por recuperar ese mercado. Los informes y comunicaciones de los agentes de Terranova en Puerto Rico revelan cómo ese mercado se transformaba entre 1950 y 1970, dejando atrás el consumo de bacalao salado y seco. Las casas comerciales en Puerto Rico hacían sus ajustes y comenzaban a importar pescado congelado o a vender ensalada ("serenata") de bacalao enlatado, un plato de la gastronomía local. Irónicamente, los informes revelan que en la década del sesenta, Puerto Rico comenzó a importar bacalao de mejor calidad, sin piel y espinas, rompiendo así con el mercado tradicional basado en bacalao de baja calidad y abriendo la puerta para importar masivamente de otros lugares como Francia, Noruega y España, países que pescaban también en las aguas de Norteamérica ${ }^{16}$. Para entonces era muy tarde pues el gusto de las familias puertorriqueñas había tomado otro rumbo.

\section{Reflexiones finales sobre la Ecología y la Economía-Mundo}

El colapso de los abastos de los gádidos en Terranova, y el cierre de esa pesquería en 1992 fue el producto de una diversidad de factores atados, en la larga duración, a la explotación de ese recurso pesquero desde que se iniciaron las incursiones pesqueras europeas en 1600, con la pesquería estacional y las plantaciones dominadas desde el West Country. En este trabajo he

\footnotetext{
16 PANL GN 31-2, Department of Natural Resources. Reports of the Fisheries Board Representatives in Puerto Rico.Varios informes de 1966. Estos informes detallan las transacciones y las opiniones de los importadores, ante la nueva clase de consumidores que había producido el proceso de industrialización en Puerto Rico.
} 
descrito parte de la densa historia de esas pesquerías y de cómo el bacalao fue un alimento barato y una mercancía producida para los mercados europeos y para los mercados caribeños y latinoamericanos para sostener la fuerza de trabajo en las plantaciones y sostener a esclavos, militares y trabajadores libres. Un alimento barato para formar parte de unas estrategias dirigidas a la reproducción de la fuerza de trabajo y la acumulación de capital (Moore, 2020, p. 95).

Esa fue su trayectoria en Puerto Rico y con el desarrollo de las centrales azucareras en el siglo $\mathrm{XX}$ y la incorporación de un proletariado rural, el bacalao mantuvo su importancia en alimentación del país, hasta los cambios provocados por el proceso de industrialización. En ese siglo, las clases comerciales de Terranova y Puerto Rico, se enfrascaron en un tête-à-tête mercantil dominado por el tema de los bajos precios impuestos por el gobierno estadounidense y las condiciones económicas de los consumidores. Como resultado, Puerto Rico fue un mercado de un gran valor para las juntas regidoras del negocio en Terranova, ya que compraba un pescado de menor calidad, que era posible producir por las condiciones de producción y reproducción de la fuerza de trabajo. En otras palabras, Puerto Rico fue el mercado más importante de Terranova durante extensos segmentos de la primera mitad de siglo $X X$, con importaciones que oscilaron entre 20 y 40 millones de libras. Cuando se escriba la historia de las poblaciones de peces y los abastos pesqueros de Terranova, habrá que contar con la participación de Puerto Rico y su rol en el proceso de intensificación de la producción, es decir, de la expansión de la frontera vertical y la horizontal. Una trayectoria de creación de valor marcada por "una dialéctica capitalizadora y reproducción apropiadora" basada en la explotación de la fuerza de trabajo y la apropiación constante de la capacidad de la naturaleza para forjar la vida (Moore, 2020, p. II7).

Los procesos descritos en este artículo han configurado (y desgastado) el oikeios del Mar del Norte, es decir, "la relación generadora y multidimensional de las especies" (Moore, 2020 p. 19), transformando la trama de la vida de los gádidos y otras especies asociadas (Davis, 20I4). No obstante, tengo muy claro que lo que hizo avanzar ese proceso de colapso lo fue el desarrollo de la flota arrastrera bajo el manto del proceso de modernización y la incursión de las flotas pesqueras europeas desde la década del cincuenta, junto a otros factores, como el cambio climático y una serie de decisiones científicas desacertadas que concluyeron en un declive del abasto (Finnlayson, 1994; Harris, 1998; Wright, 200I; Bavington, 2010; Pauly, 2019)17. No obstante, el origen de ese proceso debe examinarse con el rol de Terranova en esa economía trasatlántica, en la que Puerto Rico se insertó desde el primer día junto a otros enclaves caribeños, vinculando su historia con la de Nueva Inglaterra y sus procesos políticos y con la de Gran Bretaña, el West Country, las plantaciones en Terranova y el desarrollo de los outports. Una historia atravesada por las clases mercantiles de origen británico en St. John's y las de origen español, en San Juan; un proceso sostenido por un enlace en la Ecología-Mundo.

\section{Referencias}

Alexander, D. (1977). The Decay of the Trade:An Economic History of the Newfoundland Saltfish Trade, 1935-1965. Memorial University of Newfoundland.

\footnotetext{
17 Internacionalmente hay esfuerzos en la dirección de construir, por medio de abordajes inter y transdisciplinarios, historias ecológicas de diversos ecosistemas marinos y sus especies, tomando en consideración el consumo, la producción, el cambio climático, las transformaciones de la trama trófica y las políticas de desarrollo. Los trabajos de Pauly y MacLean, Bolster, Campling y Colas, y Davis, citados en este artículo, son ejemplos de ello.
} 
Alexander, D. (1980). The Collapse of the Saltfish Trade and Newfoundland's Integration into the North American Economy. En Hiller, J. y Neary, P. (Eds.) Newfoundlands in the Nineteenth and Twentieth Centuries: essays in interpretation (pp. 246-265). University of Toronto Press.

Antler, E. P. ( 1 982). Fisherman, Fisherwoman, Rural Proletariat: Capitalist Commodity Production in the Newfoundland Fishery. (Tesis doctoral). University of Connecticut.

Bolster, J.W. (20I2). The Mortal Sea: Fishing the Atlantic in the Age of Sail. Harvard University Press.

Braudel, F. (1972). The Mediterranean and the Mediterranean World in the Age of Phillip II. HarperCollins Publishers.

Campling, L. y Colas, A. (202I). Capitalism and the Sea:The Maritime Factor in the Making of the Modern World. Verso Books.

Cubillo de la Puente, R. (I998). El pescado en la alimentación de Castilla y León durante los siglos XVIII y XIX. Universidad de León.

Davis, R. (20I4). A Cod Forsaken Place? Fishing in an Altered State in Newfoundland. Anthropological Quarterly, 87 (3), 695-726.

Dávila-Cox, E. (1996). Este inmenso comercio: Las relaciones mercantiles entre Puerto Rico y Gran Bretaña, I844-I898. Editorial de la Universidad de Puerto Rico.

Fagan, B. (2006). Fish on Friday: Feasting, Fasting and the Discovery of the New World. Perseus Books Group.

Figueroa, L. A. (2005). Sugar, Slavery, and Freedom in Nineteenth-Century Puerto Rico. The University of North Carolina Press.

Gerhardsen, G. M. (1949). Bacalao y especies afines saladas. Estudio de la FAO sobre la Pesca. Número I.

Graffe, R. (2012). Distant Tyranny: Markets, Power, and Backwardness in Spain, 1650-I800. Princeton University Press.

Harris, M. (1998). Lament for an Ocean:The Collapse of the Atlantic Cod Fishery,ATrue Crime Story. McClelland and Stewart Inc.

Magra, C. P. (2009). The Fisherman's Cause: Atlantic Commerce and Maritime Dimensions of the American Revolution. Cambridge University Press.

Moore, J.W. (2003). Capitalism as World Ecology: Braudel and Marx on Environmental History. Organization and Environment, 16 (4), 43 I-458.

Moore, J.W. (20I3). El auge de la ecología-mundo capitalista: Las fronteras mercantiles en el auge y decadencia de la apropiación máxima. Laberinto, (38) 9-26.

Moore, J.W. (2020). El capitalismo en la trama de la vida: Ecología y acumulación de capital. Traficantes de sueños.

Neary, P. (1988). Newfoundland in the North Atlantic World, 1929-1949. McGill-Queen's University Press.

O'Leary, W. M. (198I). The Maine Sea Fisheries, 1830-1890: The Rise and Fall of a Native Industry. (Tesis doctoral). University of Maine.

Ortiz-Cuadra, C. M. (2006). Puerto Rico en la olla, jsomos aún lo que comimos? Ediciones Doce Calles, S.L.

Pauly, D. (2019). Vanishing Fish: Shifting Baselines and the Future of Global Fisheries. Greystone Books Ltd.

Pauly, D. y MacLean, J. (2003). On a Perfect Ocean:The State of Fisheries and Ecosystems in the North Atlantic Ocean. Island Press.

Pope, P. E. (2004). Fish into Wine:The Newfoundland Plantation in the Seventeenth Century. University of North Carolina Press.

Ryan,S.(1 980).The Newfoundland Salt CodTrade in the Nineteenth Century. En Hiller,J.y Neary, P. (Eds.) Newfoundlands in the Nineteenth and Twentieth Centuries: essays in interpretation (pp. 40-66). University of Toronto Press.

Sider, G. M. ( 1986). Culture and Class in Anthropology and History:A Newfoundland Illustration. Cambridge University Press.

Wright, M. (200I). A Fishery for Modern Times:The State and the Industrialization of the Newfoundland Fishery, $1934-1968$. University of Toronto Press. 


\section{RELACIONES INTERNACIONALES}

Revista académica cuatrimestral de publicación electrónica

Grupo de Estudios de Relaciones Internacionales (GERI)

Universidad Autónoma de Madrid, España

https://revistas.uam.es/relacionesinternacionales

ISSN 1699 - 3950

f facebook.com/RelacionesInternacionales

3. twitter.com/RRInternacional 